groups 4 and 5 (i.e. patients with poor PS and stage) has remained essentially stable (see figure 1).

Conclusion Our results demonstrate that survival has improved for limited groups of patients with lung cancer over the last 6 years. Stratification by clinical stage and PS has shown that survival is improving for patients with early stage cancer and good PS. Further research is required to investigate why this improvement has occurred, and to ensure all patients have equal access to advances in lung cancer care. Increasing the proportion of patients in this subgroup, with good PS and early stage disease, will also improve our survival figures.

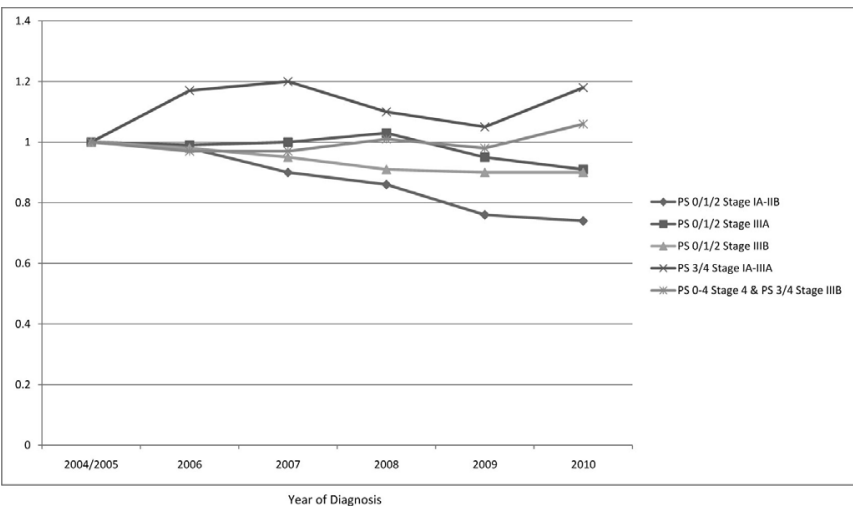

Abstract P164 Figure 1 Adjusted hazard ratios of overall survival by year

\section{P165 SURVIVAL OF PATIENTS WITH LUNG CANCER DIAGNOSED AT A TEACHING HOSPITAL IN LONDON, UK BETWEEN 2000 AND 2010}

doi:10.1136/thoraxjnl-2012-202678.226

KEN Clark, D Rao, SS Birring, R Lal, S Desai, S Pomplun, J Kelly, I Atuchar, RD Barker. Kings College Hospital, Kings Health Partners, London, United Kingdom

Background Significant resources have been applied to improving outcomes for patients with lung cancer. Here we analyse trends in survival for patients diagnosed at our institution between 2000 and 2011.

Methods All patients with suspected intra-thoracic malignancy were discussed at a weekly multi-disciplinary meeting and logged in a bespoke database. Relevant diagnostic and staging parameters were recorded. Data items were defined according to the specifications of the Lucada dataset. Patients were grouped into sequential 2 year epochs according to the date they were first seen. Analysis was restricted to the major cell types; squamous cell, adenocarcinoma, small cell and probable lung cancer unknown histology, first seen between January $1^{\text {st }} 2000$ and January $1^{\text {st }} 2010$. Survival time was censored on the $1^{\text {st }}$ September 2011. Univariate analyses with survival as the dependent variable included age group, sex, histological cell type, stage, performance status and epoch used the KaplanMeier technique. Multivariate analyses used Cox's proportional hazards model.

Results 1105 patients met the entry criteria and vital status was firmly ascertained for 1099 (99.5\%). The median age was 71 years (range 32 -96). 698 63.5\% were men. Median survival increased from 195 (95\% CI 148,242) days to 231 days (95\% CI 151,311) $\mathrm{p}<0.001$. In the $2000-1$ cohort it took 412 days for $75 \%$ of the patients to die, in 2009-10 it took 839 days. In univariate analyses there were significant differences in survival in relation to all the variables. Patients with adenocarcinoma lived longer than other cell types. In multivariate analyses age group, epoch, stage and performance status but not histology and gender remained independent predictors of survival. Histology was confounded by epoch.

Discussion These data suggest that survival for patients with lung cancer has improved over the last few years. Patients with adenocarcinoma live longer than patients with other cell types. Elsewhere we have shown that the proportion of patients with adenocarcinoma has increased in our cohort with time. Taken together these data suggest that increased longevity of lung cancer patients may be in part due to a shift towards adenocarcinoma cell type but also another factor that has occurred more recently.

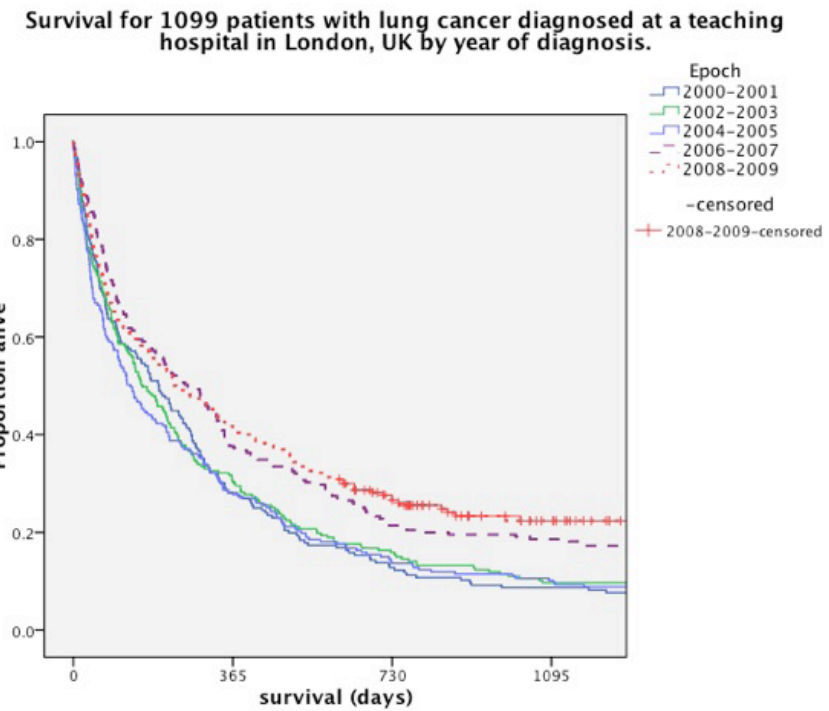

Abstract P165 Figure 1

\section{P166 ROLE OF BRONCHOSCOPY IN PATIENTS WITH HAEMOPTYSIS AND A NORMAL CT SCAN}

doi:10.1136/thoraxjnl-2012-202678.227

S Sharma, A Poorghobad, M Wood. Ashford and St Peter's Hospitals NHS Foundation Trust, Chertsey, England

Aims and Objectives To investigate the merits of conducting bronchoscopies in patients with haemoptysis and normal or nondiagnostic CT scan.

Methods Using the in-hospital bronchoscopy reporting tool, a retrospective analysis was carried out of the bronchoscopies performed on patients with haemoptysis but non-diagnostic CT scan.

Results Between September 2008 and December 2011, a total of 450 bronchoscopies were performed. After excluding the patients with CT scan abnormalities which could explain the haemoptysis, medical notes of the remaining 99 patients were analysed. Out of these, 74 patients had a significant smoking history. 79 bronchoscopes were normal with the remaining 20 examinations revealing benign pathologies. These included generalised erythema, inflammation, purulent secretions and cartilagenous nodules. No new diagnosis of malignancy was made.

Conclusions Results of our study suggest that a normal CT scan examination has a high negative predictive value for lung cancer. 
Further studies would be beneficial to ascertain the role of bronchoscopy in the investigative pathway of lung cancer in patients with normal CT scan.

Abstract P166 Table 1 Cross-Tabulation of CT and bronchoscopy results

\begin{tabular}{lcc}
\hline & Normal CT scan & Non diagnostic CT scan \\
\hline & 75 & 24 \\
Bronchoscopy results & 0 & 0 \\
Neoplasia & 12 & 10 \\
Benign & & \\
\hline
\end{tabular}

\section{P167 PATHOLOGICAL CONFIRMATION RATE OF LUNG CANCER IN ENGLAND USING THE NLCA DATABASE}

doi:10.1136/thoraxjnl-2012-202678.228

${ }^{1}$ A Khakwani, ${ }^{2}$ AL Rich, 'LJ Tata, ${ }^{3}$ HA Powell, ${ }^{4}$ RA Stanley, ${ }^{2} \mathrm{DR}$ Baldwin, ${ }^{1}$ RB Hubbard. 'Division of Epidemiology and Public Health, University of Nottingham, Nottingham, United Kingdom; ${ }^{2}$ Department of Respiratory Medicine, Nottingham University Hospital, Nottingham, United Kingdom; ${ }^{3}$ Nottingham Respiratory Research Unit, University of Nottingham, Nottingham, United Kingdom; ${ }^{4}$ Clinical Audit Support Unit, Health and Social Care Information Centre, Leeds, United Kingdom

Introduction The majority of people with lung cancer should have pathological confirmation of their diagnosis. The National Lung Cancer Audit (NLCA) recommends that NHS trusts obtain pathology (histology or cytology) for $75 \%$ of their lung cancer patients, however this figure was arbitrarily chosen and the optimal pathological confirmation rate is unknown.

The Aims of this study were to provide a simple means of benchmarking appropriate pathological confirmation rates by stratifying patients into groups, and whether obtaining pathology based on those groups is associated with a survival benefit.

Methods Using the NLCA database, we calculated the proportion of patients with non-small cell and small cell lung cancer, first seen between $1^{\text {st }}$ January 2004 and $31^{\text {st }}$ December 2010, who had pathological confirmation of their diagnosis. Using bivariate analysis, we identified the features which were most strongly associated with having pathology. We stratified our cohort according to these features and developed 4 groups (Table 1) based on the percentage of pathology obtained by the majority of NHS trusts during the study period.

Results We analysed data on 136,993 individuals. The median age at diagnosis was 72 years (IQR 64-79 years). Performance status (PS) and age were the features most strongly associated with having a pathological diagnosis. Pathological confirmation was associated with a survival benefit at 6 months for patients in groups 1-3 and at 1 year for patients in group $1 \& 2$ having adjusted for confounders (HR 0.93 \& 0.89 respectively). This survival benefit was removed when adjusted for treatment with chemotherapy (Table 2)

Discussion Stratifying by age and PS, is a simple means of benchmarking pathological confirmation rates which is more appropriate than current recommendations. We have shown better survival at six months and one year for patients who had pathological confirmation of lung cancer in groups 1 and 2 (younger patients with better PS), even after adjusting for confounders. Much of this survival advantage was accounted for by adjusting for the use of chemotherapy. We would suggest, therefore, that clinicians should aim to achieve a pathologically confirmed diagnosis in every patient in groups $1 \& 2$.
Abstract P167 Table 1 Sub groups of patients stratified by age and performance status

\begin{tabular}{ll}
\hline Group 1 & PS $0 / 1$ \& age $<65$, PS $0 / 1$ \& age $65-75$, PS 2 \& age $<65$ \\
Group 2 & PS $0 / 1$ \& age $>75$, PS $2 \&$ age $65-75$ \\
Group 3 & PS 2 \& age $>75$, PS $3 \&$ age $<65$, PS 3 \& age $65-75$ \\
Group 4 & PS $3 \&$ age $>75$, PS 4 \& age $<65$, PS 4 \& age $65-75$, PS 4 \& age $>75$
\end{tabular}

Abstract P167 Table 2 Survival within 6 months and 1 year for patients with pathological confirmation of lung cancer.

\begin{tabular}{lcccc}
\hline Survival Time & Groups & $\begin{array}{l}\text { Unadjusted HR } \\
\mathbf{( 9 5 \%} \mathbf{~ C l})\end{array}$ & Adjusted HR (95\% CI) & \\
\hline 6 months following & 1 & $0.81(0.77-0.86)$ & $0.81(0.77-0.86)$ & $1.09(1.03-1.16)$ \\
diagnosis & 2 & $0.86(0.82-0.90)$ & $0.84(0.79-0.88)$ & $1.01(0.96-1.07)$ \\
& 3 & $1.04(1.00-1.08)$ & $0.93(0.89-0.96)$ & $1.05(1.01-1.09)$ \\
& 4 & $1.14(1.10-1.18)$ & $0.98(0.95-1.02)$ & $1.05(1.01-1.09)$ \\
1 year following & 1 & $0.96(0.92-1.01)$ & $0.93(0.88-0.97)$ & $1.10(1.05-1.16)$ \\
diagnosis & 2 & $0.93(0.89-0.97)$ & $0.89(0.85-0.93)$ & $1.02(0.97-1.06)$ \\
& 3 & $1.10(1.06-1.14)$ & $0.99(0.95-1.02)$ & $1.10(1.06-1.14)$ \\
& 4 & $1.17(1.12-1.21)$ & $1.02(0.98-1.05)$ & $1.07(1.03-1.12)$ \\
\hline
\end{tabular}

${ }^{\ddagger}$ Adjusted for sex, stage, socioeconomic status, Charlson Index, ethnicity, source of referral

Adjusted for chemotherapy in addition to other variables

\section{P168 FACTORS ASSOCIATED WITH ADVANCED STAGE LUNG CANCER AT DIAGNOSIS - A RETROSPECTIVE COHORT STUDY}

doi:10.1136/thoraxjnl-2012-202678.229

E Ghorani, B O'Leary, K Asif, S Hafiz, AK Reinhardt. Whipps Cross Hospital, London, UK

Introduction Lung cancer is a major cause of mortality in the UK, with survival related to stage of disease at presentation. Better understanding of factors contributing to presentation delay might aid earlier identification and assessment of patients. Here we set out to characterise demographic features of the late presenting population at an East London hospital.

Methods Local lung cancer diagnoses between June 2005 and November $2011(n=554)$ were retrospectively determined from the National Lung Cancer Audit database. Cases for whom staging information at diagnosis was available, were selected for study $(n=460)$ and divided into those with early (stage I-IIIA, $\mathrm{n}=135$ ) and late (stage IIIB-IV, $\mathrm{n}=325$ ) disease. Socioeconomic quintiles based on patients' postcodes were defined using the Income domain of the Index of Multiple Deprivation 2010. Data were analysed by logistic regression in SPSS.

Results We found increasing age (ORs 0.95, 0.55, 0.58 for age groups $<60,60-69,70-79$ and $\geq 80$ years respectively, $p=0.027)$ to be associated with decreased risk of late presentation.

Male gender $(\mathrm{OR}=1.59,95 \% \mathrm{CI} 1.06-2.38, \mathrm{p}=0.016)$ and emergency presentation as opposed to community based referral to services $(O R=1.92,95 \%$ CI 1.19-3.10, $p=0.008)$ were associated with advanced stage disease at diagnosis.

Socioeconomic group was not found to contribute significantly to stage of disease at presentation.

Conclusion We report that age, gender but not socioeconomic status are risk factors for late stage of lung cancer at diagnosis. These findings are in line with a recent large study of the East of England cancer registry (BJC 2012; 106:1068-1075). We extend that work to show a relationship between mode of presentation and disease stage at diagnosis, in keeping with national data indicating that emergency presentation is associated with poorer survival outcomes (NCIN 2010).

In summary, this study adds to the increasing evidence that patient factors are associated with potentially avoidable late stage presentations of lung cancer, reiterating the need for targeted health promotion activities to engage at risk patient groups. 